\title{
Thyroid antibody status exerts insignificant effect on lymph node metastasis of thyroid cancer
}

\author{
Youxing Zhou ${ }^{1 \#}$, Zhiqiang Sun ${ }^{1 \#}$, Yan Zhou ${ }^{1}$, Cheng Tang ${ }^{1}$, Xiaopeng Jiang ${ }^{2}$, Fuliang Sun ${ }^{1}$, Yi Ma $^{1}$, \\ Jianfeng Cheng ${ }^{1}$
}

${ }^{1}$ Department of Surgery, JiangYuan Hospital Affiliated to Jiangsu Institute of Nuclear Medicine (Key Laboratory of Nuclear Medicine, Ministry of Health/Jiangsu Key Laboratory of Molecular Nuclear Medicine), Wuxi, China; ${ }^{2}$ Department of Information, JiangYuan Hospital Affiliated to Jiangsu Institute of Nuclear Medicine (Key Laboratory of Nuclear Medicine, Ministry of Health/Jiangsu Key Laboratory of Molecular Nuclear Medicine), Wuxi, China

Contributions: (I) Conception and design: Y Zhou, Z Sun, J Cheng; (II) Administrative support: J Cheng; (III) Provision of study materials or patients: Y Ma; (IV) Collection and assembly of data: All authors; (V) Data analysis and interpretation: Y Zhou, Z Sun, J Cheng, Y Zhou; (VI) Manuscript writing: All authors; (VII) Final approval of manuscript: All authors.

\#These authors contributed equally to this work.

Correspondence to: Dr. Jianfeng Cheng. Department of Surgery, JiangYuan Hospital Affiliated to Jiangsu Institute of Nuclear Medicine (Key Laboratory of Nuclear Medicine, Ministry of Health/Jiangsu Key Laboratory of Molecular Nuclear Medicine), 20 Qianrong Road, Wuxi 214063, China. Email: chengiianfengjsinm@163.com.

Background: To investigate whether high thyroid peroxidase antibody (TPOAb) and thyroglobulin antibody (TgAb) levels are associated with increased risk of lymph node metastasis (LNM) of thyroid cancer. Methods: Data of 2,352 patients who committed thyroidectomy from January 2018 to December 2018 at our institution were retrospectively reviewed. Of which, 806 patients diagnosed with thyroid cancer with available data of both TPOAb and TgAb were finally included, and were divided into four groups: (I) TPOAb-/TgAb- (control, n=493), (II) TPOAb+/TgAb- (n=96), (III) TPOAb-/TgAb+ (n=104), and (IV) $\mathrm{TPOAb}+/ \mathrm{TgAb}+(\mathrm{n}=113)$. The demographic and clinicopathological data were analyzed.

Results: Compared to control, significantly less extrathyroidal invasions were identified in TPOAb+ and/or $\mathrm{TgAb}+$ patients $(\mathrm{P}<0.05)$, while no significant differences for tumor size, multifocality, or central/lateral neck LNM rate were found for TPOAb+ and/or TgAb+ groups (all P>0.05). Compared to control, significantly more lymph nodes were removed during neck dissection $(\mathrm{P}<0.05)$, but there were no significant differences for the number or size of lymph nodes involved (all P>0.05) for TPOAb+ and/or TgAb+ patients. TPOAb+ and/or TgAb+ were not identified as risk factors or protect factors of LNM of thyroid cancer in Logistic regression analyses.

Conclusions: In the present study, we demonstrated that anti-thyroid peroxidase and thyroglobulin antibodies are not associated with increased risk of lymph node metastasis of thyroid cancer.

Keywords: Thyroid peroxidase antibody; thyroglobulin antibody; Hashimoto's thyroiditis; thyroid cancer; lymph node metastasis

Submitted Apr 27, 2020. Accepted for publication Sep 03, 2020.

doi: $10.21037 /$ tcr-20-1941

View this article at: http://dx.doi.org/10.21037/tcr-20-1941 


\section{Introduction}

Incidence of thyroid cancer increased rapidly worldwide in recent years, which is mainly due to early detection of thyroid nodules by imaging tools like ultrasonography $(1,2)$. Generally, when the diagnosis of thyroid cancer is established, surgery will be recommended, although active surveillance has been proposed as an alternative option for well-differentiated small volume thyroid cancer recently (2).

Regional lymph node metastases (LNM) are usually considered to a significant unfavorable factor for the prognosis for most malignant tumors, and unfortunately, are present in a large proportion of patients with thyroid cancer at the time of diagnosis $(3,4)$. However, the clinical significance of LNM and the necessity of neck dissection in thyroid cancer is still controversial $(2,5)$. The excellent outcomes of differentiated thyroid cancer observed have been argued more likely to be related to the indolent nature of the disease rather than to the effectiveness of treatment $(2,6-8)$. The value of routine prophylactic central neck dissection for such patients are conflicting, especially for small and noninvasive papillary thyroid cancer (PTC) $(2,9)$.

On one hand, in several studies, prophylactic dissection has shown no improvement in long-term patient outcome, while increasing the likelihood of temporary morbidity $(2,3,10)$. On the other hand, cervical LNM have been demonstrated in several studies to be associated with regional thyroid cancer recurrence and survival, and neck dissection is associated with better survival and a lower recurrence rate $(2,11)$. One study found that LNM significantly predicted poor overall survival outcome (12). Another study identified cervical LNM as an independent risk of decreased survival in older PTC patients (13).

There is accumulating evidence that Hashimoto's thyroiditis (HT) is associated with increased risk of thyroid cancer (14-16). While the role of HT in LNM in patients with thyroid cancer remains conflicting $(15,17,18)$. Although HT has been reported for not influencing the risk of recurrence in patients with PTC (19), there are also several studies demonstrated that HT is associated with less aggressiveness and tend to be a protective factor for LNM (20-22). At the same time, however, there are also reports that HT adversely affect the outcome of thyroid cancer, especially on the central LNM (23-25).

Thyroid peroxidase antibody (TPOAb) and thyroglobulin antibody ( $\mathrm{TgAb}$ ) are markers of immune response and important clinical markers for the diagnosis of HT. The cellular induced damage is considered to be associated with the development and possibly the progress of LNM of thyroid cancer $(26,27)$. Positive TgAb has been demonstrated to be significantly associated with LNM (23). In another study (16), TPOAb or TgAb positivity was found to be associated with more metastatic cervical lymph nodes, while combined TPOAb and TgAb positivity and single TgAb positivity were both demonstrated to be related to less distant metastasis.

The discrepancy of the previous studies might be due in part to the complexity of the role of TPOAb and TgAb in the development of HT (27). Identification of risk and protect factors of LNM will be both be helpful in the management of thyroid nodules. Therefore, the following study was undertaken to analyze the impact of high thyroid antibody levels on LNM of thyroid cancer. We present the following article in accordance with the STROBE reporting checklist (available at http://dx.doi.org/10.21037/tcr-201941).

\section{Methods}

The medical records of 2,352 consecutive patients who committed total or hemi thyroidectomy for thyroid diseases in our hospital, from the 1st of January 2018 to the 31st of December 2018, were retrospectively analyzed. Preoperative neck ultrasound, TgAb, TPOAb, and thyroid stimulating hormone (TSH), and postoperative routine pathologic examination were performed for all patients. For suspected thyroid cancer patients, surgery was based on preoperative neck ultrasound and fine-needle aspiration cytology results. Prophylactic central neck dissection was performed for almost all patients diagnosed with thyroid cancer, while only patients confirmed lateral neck LNM received lateral neck dissection. The histopathology analysis was done according to the World Health Organization Classification of Thyroid Tumors.

Preoperative serum levels of TgAb, TPOAb, and TSH at a fasting state, were measured prior to thyroid surgery at the endocrine laboratory of our institution by automated electrochemiluminescent immunoassay (Elecsys Anti-Tg, Elecsys Anti-TPO, and Elecsys TSH kits; Roche Diagnostics GmbH, Mannheim, Germany). For TgAb and TPOAb, the reference range was established as $0-115$ and $0-34 \mathrm{IU} / \mathrm{mL}$, respectively, while the minimal and maximal limit of detection was defined as $10-6,500 \mathrm{IU} / \mathrm{mL}$ and $5-600 \mathrm{IU} / \mathrm{mL}$, respectively. For TSH, the reference range was established as $0.270-4.20 \mu \mathrm{IU} / \mathrm{mL}$, while the minimal and maximal limit of detection was defined as $0.005-1,000 \mu \mathrm{IU} / \mathrm{mL}$. 


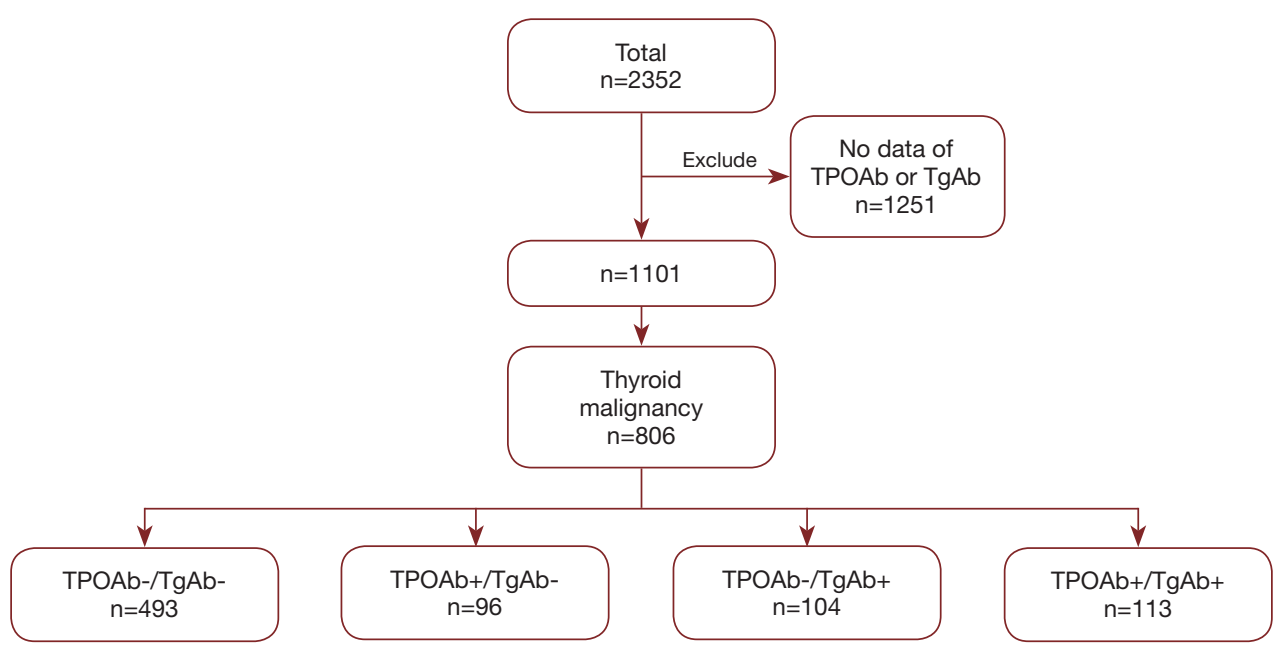

Figure 1 Flow of case selection and grouping method

Patients with no available data of TPOAb or TgAb $(\mathrm{n}=1,251)$ were excluded and the rest patients diagnosed with thyroid cancer $(n=806)$ were included into the study pool and then further grouped according to TPOAb and TgAb levels: (I) TPOAb-/TgAb- (control, $n=493)$, (II) TPOAb+/TgAb- (n=96), (III) TPOAb-/TgAb+ (n=104), and (IV) TPOAb+/TgAb+ $(\mathrm{n}=113)$ (Figure 1). TPOAb+ and TgAb+ was defined as serum TPOAb levels $>34 \mathrm{IU} / \mathrm{mL}$, and TgAb levels $>115 \mathrm{IU} / \mathrm{mL}$, respectively. The demographic and clinicopathological data of multifocality, tumor size, extrathyroidal invasion and lymph node metastasis were analyzed. All procedures performed in studies involving human participants were in accordance with the 1964 Helsinki declaration and its later amendments. The study protocol was reviewed and approved by the research ethics committee of our institution (202003). Patients' hospital records were evaluated without identification of individual patients and the authors guarantee the preservation of data and the confidentiality of the material obtained, so an informed consent did not apply for this study.

\section{Statistical analysis}

Categorical variables are presented as counts and percentages. Chi-square tests and Fisher's exact tests were utilized when appropriate. Continuous variables were presented as means \pm standard deviations, and independent $t$ tests were performed to compare the differences. Logistic regression analysis was performed to identify risk and protect factors of LNM of thyroid cancer. Variables with a $\mathrm{P}<0.100$ in the univariate analysis were considered significant and were included in the subsequent multivariate analysis. A 2 -sided $\mathrm{P}$ value $<0.05$ represented statistically significant difference. All statistical analyses were performed using SPSS 19.0 statistics software (SPSS Inc, Chicago, Illinois).

\section{Results}

\section{Demographic and clinical characteristics}

As shown in Table 1, significant female predominance was observed in all TPOAb+ and/or TgAb- patients compared to control (all $\mathrm{P}<0.001$ ). TPOAb-/TgAb+ and TPOAb+/ $\mathrm{TgAb}+$ patients were also significantly younger that control (both $\mathrm{P}<0.05$ ). As shown in Table 1, compared to control, serum levels of TSH were significantly higher for TPOAb-/ $\mathrm{TgAb}+$ patients $(\mathrm{P}=0.008)$. Compared to control, serum levels of TPOAb and TgAb were significantly higher in all $\mathrm{TPOAb}+$ and/or $\mathrm{TgAb}+$ patients (all $\mathrm{P}<0.05)$, while no significant difference was found for serum levels of TSH.

What's more, no significant difference was observed for PTC percentage or total thyroidectomy committed between $\mathrm{TPOAb}+$ and/or TgAb+ patients and control patients (all $\mathrm{P}>0.05)$.

\section{Pathological characteristics}

According to the final pathology, there were no significant differences for tumor size (mean: 8.46-9.06 vs. $9.47 \mathrm{~cm}$ ) or multifocality (all $\mathrm{P}>0.05$ ), although tumor size in TPOAb+ 
Table 1 Clinical features

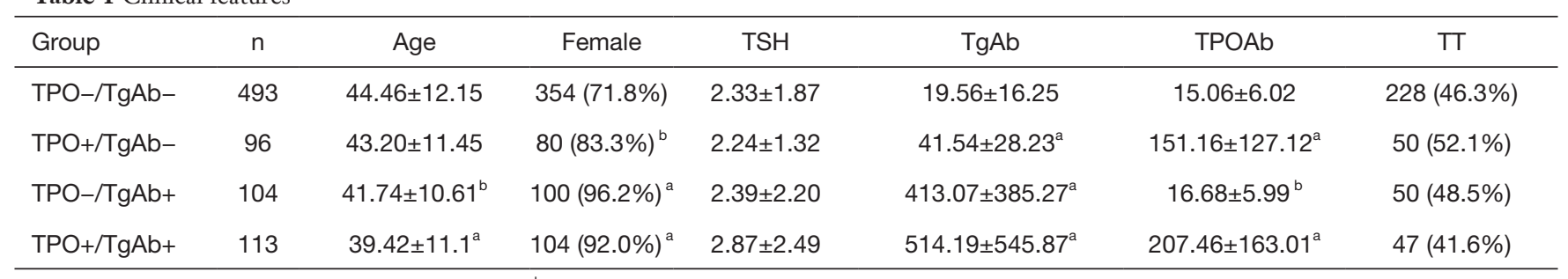

a, $\mathrm{P}<0.001$, compared to TPO-/TgAb- group; ${ }^{\mathrm{b}}, \mathrm{P}<0.05$, compared to TPO-/TgAb- group. TSH, thyroid stimulating hormone; TPOAb, thyroid peroxidase antibody; $\mathrm{TgAb}$, thyroglobulin antibody; $\mathrm{TT}$, total thyroidectomy committed.

Table 2 Pathological features

\begin{tabular}{|c|c|c|c|c|c|c|c|c|}
\hline Group & PTC & Multifocality & Tumor Size & ET & FTC & MTC & $\mathrm{HCC}$ & ATC \\
\hline TPO+/TgAb- & 94 (97.9\%) & 23 (24.2\%) & $8.46 \pm 7.29$ & $5(5.5 \%)$ & 1 & 1 & 0 & 0 \\
\hline TPO-/TgAb+ & 103 (99.0\%) & $29(28.4 \%)$ & $8.55 \pm 6.67$ & $6(6.0 \%)$ & 0 & 1 & 0 & 0 \\
\hline $\mathrm{TPO}+/ \mathrm{TgAb}+$ & $111(98.2 \%)$ & $23(20.5 \%)$ & $9.06 \pm 6.09$ & $1(0.9 \%)^{a}$ & 1 & 1 & 0 & 0 \\
\hline
\end{tabular}

a, $\mathrm{P}<0.001$, compared to TPO-/TgAb- group. TSH, thyroid stimulating hormone; TPOAb, thyroid peroxidase antibody; TgAb, thyroglobulin antibody. PTC, papillary thyroid carcinoma; ET, extrathyroidal invasion; FTC, follicular thyroid carcinoma; MTC, medullary thyroid carcinoma; HCC, Hürthle cell carcinoma; ATC, anaplastic thyroid carcinoma.

Table 3 Data of lymph node metastasis

\begin{tabular}{lccccc}
\hline Group & pN1* & pN1b* & LN number & LN metastases & LN size \\
\hline TPO-/TgAb- & $197(41.3 \%)$ & $41(8.6 \%)$ & $3.09 \pm 3.08$ & $0.74 \pm 1.34$ & $3.71 \pm 2.96$ \\
TPO+/TgAb- & $29(33.3 \%)$ & $5(5.7 \%)$ & $5.49 \pm 5.33^{\mathrm{a}}$ & $0.92 \pm 2.38^{\mathrm{b}}$ & $4.28 \pm 2.76^{\mathrm{b}}$ \\
TPO-/TgAb+ & $35(36.1 \%)$ & $11(11.3 \%)$ & $6.02 \pm 4.93^{\mathrm{a}}$ & $0.92 \pm 1.92^{\mathrm{b}}$ & $4.55 \pm 3.17^{\mathrm{b}}$ \\
TPO+/TgAb+ & $35(32.4 \%)$ & $3(2.8 \%)$ & $5.77 \pm 3.05^{\mathrm{a}}$ & $0.86 \pm 1.65^{\mathrm{b}}$ & $4.04 \pm 2.03^{\mathrm{b}}$ \\
\hline
\end{tabular}

${ }^{\mathrm{a}}, \mathrm{P}<0.001$, compared to TPO-/TgAb- group; ${ }^{\mathrm{b}}, \mathrm{P}<0.001$, compared to TPO-/TgAb- group. * , divided by the total number of patients with LN dissection. TPOAb, thyroid peroxidase antibody; TgAb, thyroglobulin antibody. LN, lymph node; LND, lymph node dissection.

and/or TgAb+ groups tend to be smaller compared to control. But, significantly less extrathyroidal invasions were identified in TPOAb-/TgAb+ group in comparison to control group $(0.9 \%$ vs. $6.0 \%, \mathrm{P}<0.001$, Table 2$)$.

\section{Comparison of LNM data}

For all 806 cases included in the study pool, 776 (96.4\%) patients underwent central neck dissection, and 187 (23.2\%) of which were bilateral; what's more, $66(8.2 \%)$ underwent lateral neck dissection and $5(0.6 \%)$ were bilateral.

As presented in Table 3, although total (N1, 32.4-36.1\% vs. $41.3 \%$ ) or lateral neck (N1b, $2.8-11.3 \%$ vs. $8.6 \%$ ) lymph node involvement tends to be less frequent for TPOAb+ and/or TgAb+ groups in comparison to control group, no significances could be detected (all $\mathrm{P}>0.05$ ). However, significantly more lymph nodes were removed during neck dissection in TPOAb+ and/or TgAb+ groups when compared to control group (all $\mathrm{P}<0.001$ ), although there were no significant differences for the number of lymph nodes involved (all $\mathrm{P}>0.05$ ). The size of lymph nodes involved tend to be larger in in TPOAb+ and/or TgAb+ groups, but the differences were not statistically significant (all $\mathrm{P}>0.05$ ).

\section{Logistic regression analyses}

To analysis the impact of TPOAb+ and/or TgAb+ status on LNM in patients with thyroid cancer, gender(male), 
Table 4 Logistic regression results

\begin{tabular}{|c|c|c|c|}
\hline & OR & $95 \% \mathrm{Cl}$ & $\mathrm{P}$ \\
\hline $\operatorname{TPOAb}(>500 \mathrm{IU} / \mathrm{mL})^{\mathrm{a}}$ & 0.682 & $0.175-2.657$ & 0.581 \\
\hline $\operatorname{TgAb}(>1,000 \mathrm{IU} / \mathrm{mL})^{\mathrm{b}}$ & 0.869 & $0.318-2.375$ & 0.784 \\
\hline TPOAb $(>34 \mathrm{IU} / \mathrm{mL})^{\mathrm{c}}$ & 0.921 & $0.846-1.004$ & 0.06 \\
\hline \multicolumn{4}{|l|}{ Multivariate } \\
\hline Age ( $\geq 55$ years) & 0.512 & $0.337-0.779$ & 0.002 \\
\hline Gender (male) & 1.674 & $1.168-2.399$ & 0.005 \\
\hline $\mathrm{TSH}$ & 1.236 & $1.096-1.393$ & 0.001 \\
\hline $\mathrm{TgAb}+/ \mathrm{TPOAb}+{ }^{\mathrm{e}}$ & 1.155 & $0.540-2.471$ & 0.711 \\
\hline
\end{tabular}

${ }^{a}$, compared to TPOAb $<500 \mathrm{IU} / \mathrm{mL} ;{ }^{b}$, compared to TgAb $<1,000 \mathrm{IU} / \mathrm{mL} ;{ }^{c}$, compared to TPOAb <34 IU/mL; ${ }^{\text {, }}$, compared to TgAb $<115 \mathrm{IU} / \mathrm{mL}$; ${ }^{e}$, reference to TgAb-/TPOAb- group. TSH, thyroid stimulating hormone; TPOAb, thyroid peroxidase antibody; TgAb, thyroglobulin antibody.

age ( $\geq 55$ years), TSH, TPOAb $(>34 \mathrm{IU} / \mathrm{mL})$, and $\mathrm{TgAb}$ $(>115 \mathrm{IU} / \mathrm{mL})$ were all subjected for univariate logistic regression analyses. What's more, TPOAb $(>500 \mathrm{IU} / \mathrm{mL})$ and $\mathrm{TgAb}(>1,000 \mathrm{IU} / \mathrm{mL})$ were also empirically included to examine the effect of extremely high levels of thyroid antibodies on LNM of thyroid cancer.

As demonstrated in Table 4, univariate logistic regression analysis revealed that for thyroid antibody levels, only TPOAb $(>34 \mathrm{IU} / \mathrm{mL})$ was included in subsequent multivariate regression analysis $(\mathrm{P}<0.1)$. Age $(\geq 55$ years $)$ was further identified as a protect factor $[\mathrm{P}=0.041, \mathrm{OR}=0.729$, 95\% confidence interval (CI): 0.538-0.987], while gender(male) $(\mathrm{OR}=1.674,95 \% \mathrm{CI}: 1.168-2.399)$, and TSH $(\mathrm{OR}=1.236$, 95\% CI: 1.096-1.393) were both identified as risk factors for $\mathrm{LNM}$ of thyroid cancer (all $\mathrm{P}<0.05$ ).

\section{Discussion}

The present study focused on and thoroughly evaluated the impact of different statuses of TPOAb+ and/or TgAb+ on LNM of thyroid cancer. In this study, we demonstrated that although $\mathrm{TPOAb}+$ and/or TgAb+ status tends to protect thyroid cancer from aggressive behaviors, especially for LNM, there were no significance detected for almost all indexes evaluated. It's not clear yet whether increasing the size of the sample will yield statistical significance, but the results of this study suggests that thyroid antibody status exerts insignificant effect on lymph node metastasis of thyroid cancer.

HT is the most prevalent autoimmune disorder characterized by the destruction of thyroid follicles caused by lymphocytes and antibody-mediated immune processes, usually accompanied by goiter and hypothyroidism $(26,28)$. Most previous studies on HT and thyroid cancer are based on results of histology, which has been recognized as the gold standard of diagnosis $(14,16)$. Unfortunately, histology can only be acquired by surgical removal of the thyroid gland and so that the risk of LNM cannot be evaluated preoperatively. What's more, the role of TPOAb and TgAb is not completely consistent in the development of $\mathrm{HT}$, and probably different as for the impact of on LNM of thyroid cancer $(26,28)$.

So, in the present study, we examined the effect of different statuses of preoperative thyroid autoantibody, $\mathrm{TPOAb}+$ and/or TgAb+, on LNM of thyroid cancer. TPOAb+ and/or TgAb+ status affect mainly young and female patients, which is consistent with previous studies based on histology results $(16,29)$. HT is a well-known for self-destruction of the thyroid gland, and may lead to hypothyroidism. In the present study, serum levels of TSH 
tend to be higher in TPOAb+ and/or TgAb+ groups, but no significance could be found. It may be due to the small number of populations studied or may also be due to thyroid hormone replacement therapy HT patients preoperatively received. Interestingly, compared to control, serum levels of TPOAb were significantly higher of TPOAb-/TgAb+ group than control group, although they were expected to be equal. Similarly, serum levels of TgAb were significantly higher in $\mathrm{TPOAb}+/ \mathrm{TgAb}$ - group B1 which suggests that there could a potential influence of each other between TPOAb and TgAb.

There are accumulating data suggesting that HT is associated with multifocality and bilaterality in thyroid cancer $(14,23,25,30,31)$. Consistent with most previous studies (16,21), significantly less extrathyroidal invasions were identified in TPOAb+/TgAb- group. But in our study, no significant differences for multifocality, tumor size, or total thyroidectomy committed could be found between the $\mathrm{TPOAb}+$ and/or TgAb+ groups and control group, which is consistent to the study of Wen et al. (27).

Due to chronic inflammation of the thyroid and surrounding tissues, significantly more lymph nodes were identified in the neck dissection specimens in TPOAb+ and/ or TgAb+ patients. Total lymph node involvement tends to be less frequent and although lymph nodes involved tend to be more and larger in TPOAb+ and/or TgAb+ groups, no significance could be detected.

Adhami et al. demonstrated that even detectable $\mathrm{TgAb}$ significantly increase the risk of LNM in PTC patients (24). Song et al. reported that positive preoperative TPOAb independently lowered the risk for structural persistent/ recurrent disease in PTC (32). TPOAb are also found to be associated with the absence of distant metastases in patients with newly diagnosed breast cancer (33). Lee et al. (17) suggested that diffuse lymphocytic infiltration was associated with aggressive features in PTC when TPOAb was negative, but tend to be indolent when TPOAb was positive. However, in the present study, both univariate and multivariate Logistic Regression analyses failed to identify the effect of TPOAb+ and/or TgAb+ status, or even extremely high levels of TPOAb or TgAb, on LNM of thyroid cancer.

Although Shen et al. (16) studied the impact of different status of high levels of TPOAb/TgAb on LNM of thyroid cancer, the population of patients they studied are limited to those subjected to radioiodine therapy. Wen et al. (27) classified patients into four groups based on thyroid antibody status, and identified TPOAb and TgAb double negative and TPOAb and TgAb double positive both as independent risk factors for central LNM in PTC, however, as it may not be reasonable to set TPOAb positive as reference.

\section{Limitations}

The retrospective design of the study, patient's selection bias and relatively small sample size. Larger prospective studies are needed to confirm results of the present study.

\section{Conclusions}

Thyroid antibody status of TPOAb+ and/or TgAb+ exerts insignificant effect on lymph node metastasis of thyroid cancer.

\section{Acknowledgments}

Funding: None.

\section{Footnote}

Reporting Checklist: The authors have completed the STROBE reporting checklist. Available at http://dx.doi. org/10.21037/tcr-20-1941

Data Sharing Statement: Available at http://dx.doi. org/10.21037/tcr-20-1941

Peer Review File: Available at http://dx.doi.org/10.21037/tcr20-1941

Conflicts of Interest: All authors have completed the ICMJE uniform disclosure form (available at http://dx.doi. org/10.21037/tcr-20-1941). The authors have no conflicts of interest to declare.

Ethical Statement: The authors are accountable for all aspects of the work in ensuring that questions related to the accuracy or integrity of any part of the work are appropriately investigated and resolved. All procedures performed in studies involving human participants were in accordance with the 1964 Helsinki declaration and its later amendments. The study protocol was reviewed and approved by the research ethics committee of our institution (202003). Patients' hospital records were evaluated without identification of individual patients and the authors 
guarantee the preservation of data and the confidentiality of the material obtained, so an informed consent did not apply for this study.

Open Access Statement: This is an Open Access article distributed in accordance with the Creative Commons Attribution-NonCommercial-NoDerivs 4.0 International License (CC BY-NC-ND 4.0), which permits the noncommercial replication and distribution of the article with the strict proviso that no changes or edits are made and the original work is properly cited (including links to both the formal publication through the relevant DOI and the license). See: https://creativecommons.org/licenses/by-nc-nd/4.0/.

\section{References}

1. Wartofsky L. Increasing world incidence of thyroid cancer: increased detection or higher radiation exposure? Hormones (Athens) 2010;9:103-8.

2. Haugen BR, Alexander EK, Bible KC, et al. 2015 American Thyroid Association Management Guidelines for Adult Patients with Thyroid Nodules and Differentiated Thyroid Cancer: The American Thyroid Association Guidelines Task Force on Thyroid Nodules and Differentiated Thyroid Cancer. Thyroid 2016;26:1-133.

3. Hughes DT, White ML, Miller BS, et al. Influence of prophylactic central lymph node dissection on postoperative thyroglobulin levels and radioiodine treatment in papillary thyroid cancer. Surgery 2010;148:1100-6.

4. Randolph GW, Duh Q, Heller KS, et al. The prognostic significance of nodal metastases from papillary thyroid carcinoma can be stratified based on the size and number of metastatic lymph nodes, as well as the presence of extranodal extension. Thyroid 2012;22:1144-52.

5. Xiao GZ, Gao L. Central Lymph Node Metastasis: Is It a Reliable Indicator of Lateral Node Involvement in Papillary Thyroid Carcinoma? World J Surg 2010;34:237-41.

6. Wang LY, Ganly I. Nodal metastases in thyroid cancer: prognostic implications and management. Future Oncol 2016;12:981-94.

7. Mazzaferri EL. Management Of Low-Risk Differentiated Thyroid Cancer. Endocr Pract 2007;13:498-512.

8. Hay ID. Management of patients with low-risk papillary thyroid carcinoma. Endocr Pract 2007;13:521-33.

9. Lan X, Sun W, Zhang H, et al. A Meta-analysis of Central Lymph Node Metastasis for Predicting Lateral
Involvement in Papillary Thyroid Carcinoma. Otolaryngol Head Neck Surg 2015;153:731-8.

10. Moreno MA, Edeikenmonroe BS, Siegel ER, et al. In Papillary Thyroid Cancer, Preoperative Central Neck Ultrasound Detects Only Macroscopic Surgical Disease, But Negative Findings Predict Excellent Long-Term Regional Control and Survival. Thyroid 2012;22:347-55.

11. Scheumann GFW, Gimm O, Wegener G, et al. Prognostic Significance and Surgical Management of Locoregional Lymph Node Metastases in Papillary Thyroid Cancer. World J Surg 1994;18:559-67.

12. Podnos YD, Smith DJ, Wagman LD, et al. The implication of lymph node metastasis on survival in patients with well-differentiated thyroid cancer. Am Surg 2005;71:731-4.

13. Zaydfudim V, Feurer ID, Griffin MR, et al. The impact of lymph node involvement on survival in patients with papillary and follicular thyroid carcinoma. Surgery 2008;144:1070-7.

14. Zhu F, Shen YB, Li FQ, et al. The Effects of Hashimoto Thyroiditis on Lymph Node Metastases in Unifocal and Multifocal Papillary Thyroid Carcinoma: A Retrospective Chinese Cohort Study. Medicine (Baltimore) 2016;95:e2674.

15. Paulson LM, Shindo ML, Schuff KG. Role of Chronic Lymphocytic Thyroiditis in Central Node Metastasis of Papillary Thyroid Carcinoma. Otolaryngol Head Neck Surg 2012;147:444-9.

16. Shen CT, Zhang X, Qiu Z, et al. Thyroid autoimmune antibodies in patients with papillary thyroid carcinoma: a double-edged sword? Endocrine 2017;58:176-83.

17. Lee YK, Park KH, Park SH, et al. Association between diffuse lymphocytic infiltration and papillary thyroid cancer aggressiveness according to the presence of thyroid peroxidase antibody and BRAF(V600E) mutation. Head Neck 2018;40:2271-9.

18. Villagelin DG, Santos RB, Romaldini JH. Is diffuse and peritumoral lymphocyte infiltration in papillary thyroid cancer a marker of good prognosis. J Endocrinol Invest 2011;34:e403-8.

19. Carvalho MS, Rosario PW, Mourão GF, et al. Chronic lymphocytic thyroiditis does not influence the risk of recurrence in patients with papillary thyroid carcinoma and excellent response to initial therapy. Endocrine 2017;55:954-8.

20. Borowczyk M, Janicki A, Dworacki G, et al. Decreased staging of differentiated thyroid cancer in patients with chronic lymphocytic thyroiditis. J Endocrinol Invest 
2019;42:45-52.

21. Dvorkin S, Robenshtok E, Hirsch D, et al. Differentiated Thyroid Cancer Is Associated With Less Aggressive Disease and Better Outcome in Patients With Coexisting Hashimotos Thyroiditis. J Clin Endocrinol Metab 2013;98:2409-14.

22. Kim EY, Kim WG, Kim WB, et al. Coexistence of chronic lymphocytic thyroiditis is associated with lower recurrence rates in patients with papillary thyroid carcinoma. Clin Endocrinol (Oxf) 2009;71:581-6.

23. Vasileiadis I, Boutzios G, Charitoudis G, et al. Thyroglobulin antibodies could be a potential predictive marker for papillary thyroid carcinoma. Ann Surg Oncol 2014;21:2725-32.

24. Adhami M, Michail P, Rao A, et al. Anti-Thyroid Antibodies and TSH as Potential Markers of Thyroid Carcinoma and Aggressive Behavior in Patients with Indeterminate Fine-Needle Aspiration Cytology. World J Surg 2020;44:363-70.

25. Iliadou PK, Effraimidis G, Konstantinos M, et al. Chronic lymphocytic thyroiditis is associated with invasive characteristics of differentiated thyroid carcinoma in children and adolescents. Eur J Endocrinol 2015;173:827-33.

26. Nagayama Y. Thyroid Autoimmunity and Thyroid Cancer - The Pathogenic Connection: A 2018 Update. Horm Metab Res 2018;50:922-31.

Cite this article as: Zhou Y, Sun Z, Zhou Y, Tang C, Jiang X, Sun F, Ma Y, Cheng J. Thyroid antibody status exerts insignificant effect on lymph node metastasis of thyroid cancer. Transl Cancer Res 2020;9(10):6423-6430. doi: 10.21037/tcr20-1941
27. Wen X, Wang B, Jin Q, et al. Thyroid Antibody Status is Associated with Central Lymph Node Metastases in Papillary Thyroid Carcinoma Patients with Hashimoto's Thyroiditis. Ann Surg Oncol 2019;26:1751-8.

28. Yoo WS, Chung HK. Recent Advances in Autoimmune Thyroid Diseases. Endocrinol Metab (Seoul) 2016;31:379-85.

29. Liang J, Zeng W, Fang F, et al. Clinical analysis of Hashimoto thyroiditis coexistent with papillary thyroid cancer in 1392 patients. Acta Otorhinolaryngol Ital 2017;37:393-400.

30. Dong S, Xie XJ, Xia Q, et al. Indicators of multifocality in papillary thyroid carcinoma concurrent with Hashimoto's thyroiditis. Am J Cancer Res 2019;9:1786-95.

31. Dong S, Xia Q, Wu YJ. High TPOAb Levels (>1300 IU/ $\mathrm{mL}$ ) Indicate Multifocal PTC in Hashimoto's Thyroiditis Patients and Support Total Thyroidectomy. Otolaryngol Head Neck Surg 2015;153:20-6.

32. Song E, Oh HS, Jeon MJ, et al. The value of preoperative antithyroidperoxidase antibody as a novel predictor of recurrence in papillary thyroid carcinoma. Int J Cancer 2019;144:1414-20.

33. Farahati J, Roggenbuck D, Gilman E, et al. Anti-thyroid peroxidase antibodies are associated with the absence of distant metastases in patients with newly diagnosed breast cancer. Clin Chem Lab Med 2012;50:709-14. 